\title{
Global weak solutions to a 3-dimensional degenerate and singular chemotaxis-Navier-Stokes system with logistic source
}

\author{
Shunsuke Kurima \\ Department of Mathematics, Tokyo University of Science \\ 1-3, Kagurazaka, Shinjuku-ku, Tokyo 162-8601, Japan \\ shunsuke.kurima@gmail.com \\ Masaaki Mizukami*† \\ Department of Mathematics, Tokyo University of Science \\ 1-3, Kagurazaka, Shinjuku-ku, Tokyo 162-8601, Japan \\ masaaki.mizukami.math@gmail.com
}

October 9, 2018

\begin{abstract}
This paper considers the degenerate and singular chemotaxis-Navier-Stokes system with logistic term

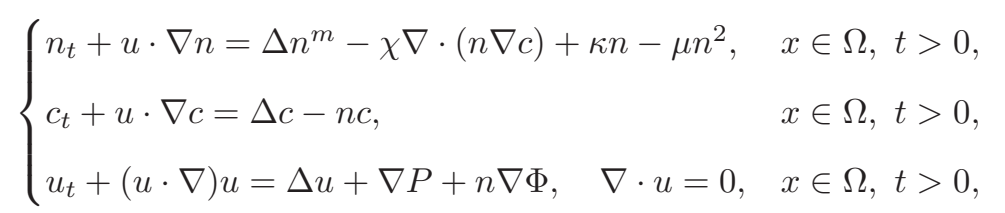

where $\Omega \subset \mathbb{R}^{3}$ is a bounded domain and $\chi, \kappa \geq 0$ and $m, \mu>0$. In the above system without fluid environment Jin [11] showed existence and boundedness of global weak solutions. On the other hand, in the above system with $m=1$, Lankeit [14] established global existence of weak solutions. However, the above system with $m>0$ has not been studied yet. The purpose of this talk is to establish global existence of weak solutions in the chemotaxis-Navier-Stokes system with degenerate diffusion and logistic term.
\end{abstract}

2010Mathematics Subject Classification. Primary: 35K55; Secondary: 92C17; 35Q35.

Key words and phrases: chemotaxis-Navier-Stokes system; degenerate diffusion; global existence.

*Partially supported by JSPS Research Fellowships for Young Scientists (No. 17J00101).

${ }^{\dagger}$ Corresponding author 


\section{Introduction and results}

In the study of partial differential equations mathematical models describing the natural phenomena, e.g., the heat equation, the Fisher-KPP equation and so on, are one of the important topics in the mathematical analysis, and studied by many mathematicians. Recently, there have been many variations of systems of partial differential equations which describe complicated phenomena. One of systems which describe important biological phenomena related to animals life is the Keller-Segel system

$$
\begin{aligned}
n_{t} & =\Delta n^{m}-\chi \nabla \cdot\left(n^{q-1} \nabla c\right), \\
c_{t} & =\Delta c-c+n, \quad x \in \Omega, t>0,
\end{aligned}
$$

where $\Omega \subset \mathbb{R}^{N}(N \in \mathbb{N}), m>0, \chi \geq 0, q \geq 2$, which describes migration of species by chemotaxis which is the property such that species move towards higher concentration of the chemical substance. Here $\Delta n^{m}$ with $m=1$, that is, $\Delta n$ is called a linear diffusion and $\Delta n^{m}$ with $m \neq 1$ is called a nonlinear diffusion. In other words, the case that $m>1$ is said to be a degenerate diffusion or a porous medium diffusion, and the case that $0<m<1$ is said to be a singular diffusion or a fast diffusion. This system with $m=1$ and $q=2$ was first proposed by Keller-Segel $[\mathbf{1 2}]$ and then the system with $m, q \in \mathbb{R}$ was suggested by Hillen-Painter [6]. In the case that $m=1$ and $q=2$ it is known that the size of initial data determines behaviour of solutions to the 2-dimensional Keller-Segel system. More precisely, there exists some constant $C>0$ such that if an initial data $n_{0}$ satisfies $\left\|n_{0}\right\|_{L^{1}(\Omega)}<C$ then global bounded classical solutions exist $([\mathbf{1 7}])$, moreover, for any $m>C$ there exist initial data such that $\left\|n_{0}\right\|_{L^{1}(\Omega)}=m$ and the corresponding solution blows up in finite time $([8,16])$, where $C>0$ can be given as $C=\frac{8 \pi}{\chi}$ in the radial setting and $C=\frac{4 \pi}{\chi}$ in the nonradial setting.

In the other dimensional case we can see that there are many global/blow-up solutions. In the case that $m=1, q=2$ and $N=1$ Osaki-Yagi [19] showed that global existence and boundedness hold for all smooth initial data: This means that there is not a blow-up solution in the 1-dimensional setting. On the other hand, it is known that the 3-dimensional case has many blow-up solutions: Winkler [27] established that for all $m>0$ there are initial data $n_{0}$ such that $\left\|n_{0}\right\|_{L^{1}(\Omega)}=m$ and the corresponding solution blows up in finite time. To obtain global existence of classical solutions we need some additional conditions for initial data: Winkler [24] and Cao [1] proved existence of global boundedness classical solutions under conditions that initial data $\left(n_{0}, c_{0}\right)$ is small enough with respect to a suitable Lebesgue norm.

On the other hand, in the case that $m \geq 1$ and $q \geq 2$, it is known that relations between $m$ and $q$ determine whether solutions of the Keller-Segel system exist globally

or not; in the case that $m>q-\frac{2}{N}$ Ishida-Seki-Yokota [9] obtained global existence of solutions; on the other hand, in the case that $m<q-\frac{2}{N}$ Ishida-Yokota [10] proved that there exist initial data such that the corresponding solution blows up in finite or infinite time, and recently Hashira-Ishida-Yokota [5] found initial data such that the solution blows up in finite time.

As a generalized problem, the nonlinear Keller-Segel system with logistic source which 
the first equation in the above system is replaced with

$$
n_{t}=\Delta n^{m}-\chi \nabla \cdot\left(n^{q-1} \nabla c\right)+\kappa n-\mu n^{2},
$$

where $m, \mu>0, \chi, \kappa \geq 0, q \geq 2$, was also studied, and it is shown that the logistic source $\kappa n-\mu n^{2}$ suppresses blow-up phenomenon. When $m=1$ and $q=2$, in the 2dimensional setting Osaki et al. [18] obtained global existence and boundedness for all smooth initial data, and in the higher dimensional setting Winkler [25] proved existence of global classical solutions under largeness conditions for $\mu>0$; in the Keller-Segel system with logistic source global existence of solutions holds even though the $L^{1}$-norm of the initial data is large enough. Moreover, Lankeit [13] established global existence of weak solutions without largeness condition for $\mu>0$. On the other hand, in the case that $q=2$ a resent result established by Zheng-Wang [30] asserted that the condition that $m>1+\frac{(N-2)_{+}}{N+2}$ derives global existence and boundedness.

As we confirmed before, in the Keller-Segel system the relation between $m$ and $q$ strongly affects behaviour of solutions, and the logistic source often relaxes conditions for global existence. Thus "what is the condition for $m$ to derive existence of global/blow-up solutions?" makes one of the main topics. This will be also one of the important topics in the study of the chemotaxis-(Navier-)Stokes system with logistic source

$$
\begin{aligned}
n_{t}+u \cdot \nabla n & =\Delta n^{m}-\chi \nabla \cdot\left(n^{q-1} \nabla c\right)+\kappa n-\mu n^{2}, \\
c_{t}+u \cdot \nabla c & =\Delta c-n c, \\
u_{t}+\lambda(u \cdot \nabla) u & =\Delta u+\nabla P+n \nabla \Phi, \nabla \cdot u=0, \quad x \in \Omega, t>0,
\end{aligned}
$$

where $m, \mu>0, \chi, \kappa \geq 0, q \geq 2, \lambda=0$ (the chemotaxis-Stokes system) or $\lambda=1$ (the chemotaxis-Navier-Stokes system). In the case that $m=1, q=2$ and moreover $\kappa=\mu=0$ Winkler established global existence of classical solutions in the 2-dimensional setting $([\mathbf{2 6}])$ and obtained existence of global weak solutions in the 3-dimensional setting ([29]). In the case that $q=2$ and $\kappa=\mu=0$ Tao-Winkler [23] and Chung-Kang [3] showed global existence under the condition that $m>1$.

On the other hand, in the chemotaxis-(Navier-)Stokes system with logistic source results similar to those in the Keller-Segel system with logistic source hold. In the case of the fluid-free system Lankeit-Wang [15] showed global existence and boundedness in the system with $m=1$ under some largeness conditions for $\mu>0$, and Jin [11] proved global existence of weak solutions of the system with $m>1$. In the system with fluid equation and $m=1, q=2$ global existence and boundedness of classical solutions hold in the 2-dimensional setting (cf. [7]) and existence of global bounded classical solutions to the chemotaxis-Stokes system holds under some largeness conditions for $\mu>0$ in the 3-dimensional setting (cf. [2]), and moreover Lankeit [14] showed that global existence of weal solutions in the system without largeness conditions for $\mu>0$. However, the chemotaxis-Navier-Stokes system with degenerate diffusion and logistic source has never been tried; which means that conditions of $m$ for global existence of weak solutions are still open problem. The purpose of this paper is to derive some condition of $m$ for existence of global weak solutions in the degenerate chemotaxis-Navier-Stokes system with logistic 
source. Here we note that the methods in [11] cannot be applied to this problem because of the difficulty of the Navier-Stokes equation (explained later).

In order to attain this purpose we consider the following degenerate and singular chemotaxis-Navier-Stokes system with logistic term:

$$
\begin{cases}n_{t}+u \cdot \nabla n=\Delta n^{m}-\chi \nabla \cdot(n \nabla c)+\kappa n-\mu n^{2}, & x \in \Omega, t>0, \\ c_{t}+u \cdot \nabla c=\Delta c-n c, & x \in \Omega, t>0, \\ u_{t}+(u \cdot \nabla) u=\Delta u+\nabla P+n \nabla \Phi, \quad \nabla \cdot u=0, & x \in \Omega, t>0, \\ \partial_{\nu} n^{m}=\partial_{\nu} c=0, \quad u=0, & x \in \partial \Omega, t>0, \\ n(x, 0)=n_{0}(x), c(x, 0)=c_{0}(x), u(x, 0)=u_{0}(x), & x \in \Omega,\end{cases}
$$

where $\Omega$ is a bounded domain in $\mathbb{R}^{3}$ with smooth boundary $\partial \Omega$ and $\partial_{\nu}$ denotes differentiation with respect to the outward normal of $\partial \Omega ; \chi, \kappa \geq 0$ and $\mu, m>0$ are constants; $n_{0}, c_{0}, u_{0}, \Phi$ are known functions satisfying

$$
\begin{aligned}
& 0<n_{0} \in C(\bar{\Omega}), \quad 0<c_{0} \in W^{1, q}(\Omega), \quad u_{0} \in D\left(A^{\theta}\right), \\
& \Phi \in C^{1+\beta}(\bar{\Omega})
\end{aligned}
$$

for some $q>3, \theta \in\left(\frac{3}{4}, 1\right), \beta>0$ and $A$ denotes the realization of the Stokes operator under homogeneous Dirichlet boundary conditions in the solenoidal subspace $L_{\sigma}^{2}(\Omega)$ of $L^{2}(\Omega)$.

Before stating the main theorem, we define weak solutions of (1.1).

Definition 1.1. A triplet $(n, c, u)$ is called a (global) weak solution of (1.1) if

$$
\begin{aligned}
n & \in L_{\mathrm{loc}}^{2}\left([0, \infty) ; L^{2}(\Omega)\right), \\
n^{m} & \in L_{\mathrm{loc}}^{\frac{4}{3}}\left([0, \infty) ; W^{1, \frac{4}{3}}(\Omega)\right), \\
c & \in L_{\mathrm{loc}}^{2}\left([0, \infty) ; W^{1,2}(\Omega)\right), \\
u & \in L_{\mathrm{loc}}^{2}\left([0, \infty) ; W_{0, \sigma}^{1,2}(\Omega)\right)
\end{aligned}
$$

and the identities

$$
\begin{aligned}
& -\int_{0}^{\infty} \int_{\Omega} n \varphi_{t}-\int_{\Omega} n_{0} \varphi(\cdot, 0)-\int_{0}^{\infty} \int_{\Omega} n u \cdot \nabla \varphi \\
& =-\int_{0}^{\infty} \int_{\Omega} \nabla n^{m} \cdot \nabla \varphi+\chi \int_{0}^{\infty} \int_{\Omega} n \nabla c \cdot \nabla \varphi+\int_{0}^{\infty} \int_{\Omega}\left(\kappa n-\mu n^{2}\right) \varphi \\
& -\int_{0}^{\infty} \int_{\Omega} c \varphi_{t}-\int_{\Omega} c_{0} \varphi(\cdot, 0)-\int_{0}^{\infty} \int_{\Omega} c u \cdot \nabla \varphi=-\int_{0}^{\infty} \int_{\Omega} \nabla c \cdot \nabla \varphi-\int_{0}^{\infty} \int_{\Omega} n c \varphi, \\
& -\int_{0}^{\infty} \int_{\Omega} u \cdot \psi_{t}-\int_{\Omega} u_{0} \cdot \psi(\cdot, 0)-\int_{0}^{\infty} \int_{\Omega} u \otimes u \cdot \nabla \psi \\
& =-\int_{0}^{\infty} \int_{\Omega} \nabla u \cdot \nabla \psi+\int_{0}^{\infty} \int_{\Omega} n \nabla \psi \cdot \nabla \Phi
\end{aligned}
$$

hold for all $\varphi \in C_{0}^{\infty}(\bar{\Omega} \times[0, \infty))$ and all $\psi \in C_{0, \sigma}^{\infty}(\Omega \times[0, \infty))$, respectively. 
The main result reads as follows. The following theorem gives existence of global weak solutions to (1.1).

Theorem 1.1. Let $\Omega \subset \mathbb{R}^{3}$ be a bounded smooth domain and let $\chi, \kappa \geq 0$ and $\mu, m>0$. Assume that $n_{0}, c_{0}, u_{0}$ and $\Phi$ satisfy $(1.2)-(1.3)$ with some $q>3, \theta \in\left(\frac{3}{4}, 1\right)$ and $\beta \in(0,1)$. Then there exists a weak solution of (1.1), which can be approximated by a sequence of solutions $\left(n_{\varepsilon}, c_{\varepsilon}, u_{\varepsilon}\right)$ of an approximate problem (see Section 2) in a pointwise manner.

Remark 1.1. This result means existence of weak solutions to (1.1) for all $m>0$; which implies that we could construct weak solutions of (1.1) in not only the case that $m>1$ (the case of a porous medium diffusion) but also $0<m<1$ (the case of a fast diffusion).

The proof of Theorem 1.1 can be applied to a nondegenerate chemotaxis-Navier-Stokes system which namely is the case that $\Delta n^{m}$ is replaced with $\Delta(n+1)^{m}$, and enables us to see the following result.

Corollary 1.2. Let $\Omega \subset \mathbb{R}^{3}$ be a bounded smooth domain and let $\chi, \kappa \geq 0$ and $\mu, m>0$. Assume that $n_{0}, c_{0}, u_{0}$ and $\Phi$ satisfy $(1.2)-(1.3)$ with some $q>3, \theta \in\left(\frac{3}{4}, 1\right)$ and $\beta \in(0,1)$. Then there exists a weak solution of the nondegenerate chemotaxis-Navier-Stokes system.

The strategy for the proof of Theorem 1.1 is described as follows. We start with the construction of local approximate solutions of (1.1). We next derive estimates for the approximate solution. Thanks to the estimates, we extend the local approximate solution globally in time. Finally, passing to the limit from the global approximate solution, we obtain the desired global weak solution. In the previous work by Jin [11] which deals with the fluid-free case, aided by uniform $L^{\infty}$-estimates, we can attain convergences of approximate solutions. Now, because of the difficulty of the Navier-Stokes equation, we could not expect $L^{\infty}$-boundedness of solutions; thus we have to need different methods to consider this problem. More precisely, we rely on the Lions-Aubin lemma. In this strategy the key is to establish estimates for $\nabla n_{\varepsilon}$, where $n_{\varepsilon}$ is the solution of the first equation in the approximate problem and $\varepsilon$ is a positive parameter. More precisely, generalizing calculations in [14], we first obtain

$$
\int_{0}^{T} \int_{\Omega} \frac{\left(n_{\varepsilon}+\varepsilon\right)^{m-1}\left|\nabla n_{\varepsilon}\right|^{2}}{n_{\varepsilon}}=\left(\frac{2}{m+1}\right)^{2} \int_{0}^{T} \int_{\Omega} \frac{\left|\nabla\left(n_{\varepsilon}+\varepsilon\right)^{\frac{m+1}{2}}\right|^{2}}{n_{\varepsilon}} \leq C_{1}(T)
$$

for all $\varepsilon \in(0,1)$ with some constant $C_{1}(T)>0$. Aided by this estimate we can have that $\left(\left(n_{\varepsilon}+\varepsilon\right)^{\frac{m+1}{2}}\right)_{\varepsilon \in(0,1)}$ is bounded in $L^{\frac{4}{3}}\left([0, T) ; W^{1, \frac{4}{3}}(\Omega)\right)$; however, it seems to be difficult to obtain the estimate for $\partial_{t}\left(n_{\varepsilon}+\varepsilon\right)^{\frac{m+1}{2}}$ for all $m>0$. Thus we need additional estimates for approximate solutions. Here the inequality (1.4) ensures that

$$
\int_{0}^{T} \int_{\Omega}\left(n_{\varepsilon}+\varepsilon\right)^{m-2}\left|\nabla n_{\varepsilon}\right|^{2} \leq \int_{0}^{T} \int_{\Omega} \frac{\left(n_{\varepsilon}+\varepsilon\right)^{m-1}\left|\nabla n_{\varepsilon}\right|^{2}}{n_{\varepsilon}} \leq C_{1}(T)
$$

for all $\varepsilon \in(0,1)$. This together with the identity

$$
\int_{0}^{T} \int_{\Omega}\left|\nabla\left(n_{\varepsilon}+\varepsilon\right)^{\frac{m}{2}}\right|^{2}=\frac{m^{2}}{4} \int_{0}^{T} \int_{\Omega}\left(n_{\varepsilon}+\varepsilon\right)^{m-2}\left|\nabla n_{\varepsilon}\right|^{2}
$$


means that $\left(\left(n_{\varepsilon}+\varepsilon\right)^{\frac{m}{2}}\right)_{\varepsilon \in(0,1)}$ is bounded in $L_{\mathrm{loc}}^{2}\left([0, \infty) ; W^{1,2}(\Omega)\right)$. We moreover see that

$$
\left\|\partial_{t}\left(n_{\varepsilon}+\varepsilon\right)^{\frac{m}{2}}\right\|_{L^{1}\left(0, T ;\left(W_{0}^{2,4}(\Omega)\right)^{*}\right)} \leq C_{2}(T)
$$

for all $\varepsilon \in(0,1)$ with some $C_{2}(T)>0$, which derives that $\left(\partial_{t}\left(n_{\varepsilon}+\varepsilon\right)^{\frac{m}{2}}\right)_{\varepsilon \in(0,1)}$ is bounded in $L^{1}\left(0, T ;\left(W_{0}^{2,4}(\Omega)\right)^{*}\right)$. Then, aided by the Lions-Aubin theorem, we can show convergences of solutions of the approximation of (1.1) and we can prove Theorem 1.1.

The plan of this paper is as follows. In Section 2 we introduce the approximate problem (2.1) of (1.1) and establish global existence in (2.1). In Section 3 we show the several estimates for solutions to the approximate problem of (1.1). Finally, in Section 4 we prove Theorem 1.1 by passage to the limit in the approximate problem via estimates from Section 3.

\section{Global existence in an approximate problem}

We start by considering the following approximate problem with parameter $\varepsilon>0$ :

$$
\left\{\begin{array}{l}
n_{\varepsilon t}+u_{\varepsilon} \cdot \nabla n_{\varepsilon}=\Delta\left(n_{\varepsilon}+\varepsilon\right)^{m}-\chi \nabla \cdot\left(\frac{n_{\varepsilon}}{1+\varepsilon n_{\varepsilon}} \nabla c_{\varepsilon}\right)+\kappa n_{\varepsilon}-\mu n_{\varepsilon}^{2}, \\
c_{\varepsilon t}+u_{\varepsilon} \cdot \nabla c_{\varepsilon}=\Delta c_{\varepsilon}-c_{\varepsilon} \frac{1}{\varepsilon} \log \left(1+\varepsilon n_{\varepsilon}\right), \\
u_{\varepsilon t}+\left(Y_{\varepsilon} u_{\varepsilon} \cdot \nabla\right) u_{\varepsilon}=\Delta u_{\varepsilon}+\nabla P_{\varepsilon}+n_{\varepsilon} \nabla \Phi, \quad \nabla \cdot u_{\varepsilon}=0, \\
\left.\partial_{\nu} n_{\varepsilon}\right|_{\partial \Omega}=\left.\partial_{\nu} c_{\varepsilon}\right|_{\partial \Omega}=0,\left.\quad u_{\varepsilon}\right|_{\partial \Omega}=0, \\
n_{\varepsilon}(\cdot, 0)=n_{0}, \quad c_{\varepsilon}(\cdot, 0)=c_{0}, \quad u_{\varepsilon}(\cdot, 0)=u_{0},
\end{array}\right.
$$

where $Y_{\varepsilon}=(1+\varepsilon A)^{-1}$. In this section we shall show global existence of solutions to the approximate problem (2.1). We first give the following result which states local existence in (2.1).

Lemma 2.1. Let $\chi, \kappa \geq 0, \mu>0, m>0$ and let $\Phi \in C^{1+\beta}(\bar{\Omega})$ for some $\beta \in(0,1)$. Assume that $n_{0}, c_{0}, u_{0}$ satisfy (1.2) with some $q>3, \theta \in\left(\frac{3}{4}, 1\right)$. Then for each $\varepsilon>0$ there exist $T_{\max , \varepsilon}$ and uniquely determined functions:

$$
\begin{aligned}
n_{\varepsilon} & \in C^{0}\left(\bar{\Omega} \times\left[0, T_{\max , \varepsilon}\right)\right) \cap C^{2,1}\left(\bar{\Omega} \times\left(0, T_{\max , \varepsilon}\right)\right), \\
c_{\varepsilon} & \in C^{0}\left(\bar{\Omega} \times\left[0, T_{\max , \varepsilon}\right)\right) \cap C^{2,1}\left(\bar{\Omega} \times\left(0, T_{\max , \varepsilon}\right)\right) \cap L_{\mathrm{loc}}^{\infty}\left(\left[0, T_{\max , \varepsilon}\right) ; W^{1, q}(\Omega)\right), \\
u_{\varepsilon} & \in C^{0}\left(\bar{\Omega} \times\left[0, T_{\max , \varepsilon}\right)\right) \cap C^{2,1}\left(\bar{\Omega} \times\left(0, T_{\max , \varepsilon}\right)\right),
\end{aligned}
$$

which together with some $P_{\varepsilon} \in C^{1,0}\left(\bar{\Omega} \times\left(0, T_{\max , \varepsilon}\right)\right)$ solve $(2.1)$ classically. Moreover, $n_{\varepsilon}$ and $c_{\varepsilon}$ are positive and the following alternative holds: $T_{\max , \varepsilon}=\infty$ or

$$
\left\|n_{\varepsilon}(\cdot, t)\right\|_{L^{\infty}(\Omega)}+\left\|c_{\varepsilon}(\cdot, t)\right\|_{W^{1, q}(\Omega)}+\left\|A^{\theta} u_{\varepsilon}(\cdot, t)\right\|_{L^{2}(\Omega)} \rightarrow \infty
$$

as t $\nearrow T_{\max , \varepsilon}$.

Proof. This lemma can be shown by a standard fixed point theorem with parabolic regularity arguments. More precisely, a combination of the proofs of [21, Lemma 2.1] and [26, Lemma 2.1] enables us to obtain local existence in (2.1). 
In the following for all $\varepsilon \in(0,1)$ we denote by $\left(n_{\varepsilon}, c_{\varepsilon}, u_{\varepsilon}\right)$ the corresponding solution of (2.1) given by Lemma 2.1 and by $T_{\max , \varepsilon}$ its maximal existence time. Then we shall see global existence of solutions to the approximate problem (2.1) and their useful estimates. We first recall basic inequalities which are often used in studies of the chemotaxis-NavierStokes system.

Lemma 2.2. There exists a constant $C_{1}>0$ such that

$$
\int_{\Omega} n_{\varepsilon}(\cdot, t) \leq C_{1} \quad \text { for all } t \in\left(0, T_{\max , \varepsilon}\right) \text { and all } \varepsilon>0 .
$$

Moreover, there exists $C_{2}>0$ satisfying

$$
\int_{t}^{t+\tau} \int_{\Omega} n_{\varepsilon}^{2} \leq C_{2}
$$

holds for all $t \in\left(0, T_{\max , \varepsilon}-\tau\right)$ and all $\varepsilon>0$, where $\tau \in\left(0, T_{\max , \varepsilon}\right)$.

Proof. Integrating the first equation in (2.1) shows this lemma.

Lemma 2.3. The function $t \mapsto\left\|c_{\varepsilon}(\cdot, t)\right\|_{L^{\infty}(\Omega)}$ is nonincreasing. In particular,

$$
\left\|c_{\varepsilon}(\cdot, t)\right\|_{L^{\infty}(\Omega)} \leq\left\|c_{0}\right\|_{L^{\infty}(\Omega)}
$$

holds for all $t \in\left(0, T_{\max , \varepsilon}\right)$ and all $\varepsilon>0$. Moreover, we have

$$
\int_{0}^{T_{\max , \varepsilon}} \int_{\Omega}\left|\nabla c_{\varepsilon}\right|^{2} \leq \frac{1}{2} \int_{\Omega}\left|c_{0}\right|^{2} \quad \text { for all } \varepsilon>0 .
$$

Proof. Applying the maximum principle to the second equation in (2.1) (see e.g., [28, Lemma 2.1]), we can establish the $L^{\infty}$-estimate for $c_{\varepsilon}$. Moreover, multiplying the third equation in $(2.1)$ by $c_{\varepsilon}$ and integrating it over $\Omega \times\left(0, T_{\max , \varepsilon}\right)$ imply this lemma.

We next consider an estimate for the energy function $\mathcal{F}_{\varepsilon}:\left(0, T_{\max , \varepsilon}\right) \rightarrow \mathbb{R}$ defined as

$$
\mathcal{F}_{\varepsilon}(t):=\int_{\Omega} n_{\varepsilon}(\cdot, t) \log n_{\varepsilon}(\cdot, t)+\frac{\chi}{2} \int_{\Omega} \frac{\left|\nabla c_{\varepsilon}(\cdot, t)\right|^{2}}{c_{\varepsilon}(\cdot, t)}+K \chi \int_{\Omega}\left|u_{\varepsilon}(\cdot, t)\right|^{2}
$$

with some $K>0$, which plays an important role not only in the case that $m=1([\mathbf{1 4}])$ but also in the case that $m>0$. In order to see an estimate for $\mathcal{F}_{\varepsilon}$ we provide the following 3 lemmas.

Lemma 2.4. There exists a constant $C>0$ such that for all $\varepsilon>0$,

$$
\frac{d}{d t} \int_{\Omega} n_{\varepsilon} \log n_{\varepsilon}+\frac{\mu}{2} \int_{\Omega} n_{\varepsilon}^{2} \log n_{\varepsilon}+\frac{4 m}{(m+1)^{2}} \int_{\Omega} \frac{\left|\nabla\left(n_{\varepsilon}+\varepsilon\right)^{\frac{m+1}{2}}\right|^{2}}{n_{\varepsilon}} \leq \chi \int_{\Omega} \frac{\nabla n_{\varepsilon} \cdot \nabla c_{\varepsilon}}{1+\varepsilon n_{\varepsilon}}+C
$$

on $\left(0, T_{\max , \varepsilon}\right)$. 
Proof. We first obtain from $\nabla \cdot u_{\varepsilon}=0$ in $\Omega \times\left(0, T_{\max , \varepsilon}\right)$ and straightforward calculations that

$$
\begin{aligned}
\frac{d}{d t} \int_{\Omega} n_{\varepsilon} \log n_{\varepsilon}= & \int_{\Omega} \log n_{\varepsilon} \Delta\left(n_{\varepsilon}+\varepsilon\right)^{m}-\chi \int_{\Omega} \log n_{\varepsilon} \nabla \cdot\left(\frac{n_{\varepsilon}}{1+\varepsilon n_{\varepsilon}} \nabla c_{\varepsilon}\right) \\
& +\kappa \int_{\Omega} n_{\varepsilon} \log n_{\varepsilon}-\mu \int_{\Omega} n_{\varepsilon}^{2} \log n_{\varepsilon}+\kappa \int_{\Omega} n_{\varepsilon}-\mu \int_{\Omega} n_{\varepsilon}^{2}
\end{aligned}
$$

Then, noting from the boundedness of the functions $s \mapsto\left(\kappa s-\frac{\mu}{2} s^{2}\right) \log s+\kappa s-\mu s^{2}$ on $(0, \infty)$ that

$$
\kappa \int_{\Omega} n_{\varepsilon} \log n_{\varepsilon}-\frac{\mu}{2} \int_{\Omega} n_{\varepsilon}^{2} \log n_{\varepsilon}+\kappa \int_{\Omega} n_{\varepsilon}-\mu \int_{\Omega} n_{\varepsilon}^{2} \leq C_{1}
$$

with some $C_{1}>0$, we can see from (2.2) with the relation

$$
\begin{aligned}
\int_{\Omega} \log n_{\varepsilon} \Delta\left(n_{\varepsilon}+\varepsilon\right)^{m} & =-m \int_{\Omega}\left(n_{\varepsilon}+\varepsilon\right)^{m-1} \frac{\left|\nabla n_{\varepsilon}\right|^{2}}{n_{\varepsilon}} \\
& =-\frac{4 m}{(m+1)^{2}} \int_{\Omega} \frac{\left|\nabla\left(n_{\varepsilon}+\varepsilon\right)^{\frac{m+1}{2}}\right|^{2}}{n_{\varepsilon}}
\end{aligned}
$$

that this lemma holds.

The following 2 lemmas have already been proved in the proofs of $[\mathbf{1 4}$, Lemmas 2.8 and 2.9]. Thus we only recall statements of lemmas.

Lemma 2.5. There exist $K, C, k>0$ such that for all $\varepsilon>0$,

$$
\begin{aligned}
\frac{d}{d t} \int_{\Omega} \frac{\left|\nabla c_{\varepsilon}\right|^{2}}{c_{\varepsilon}} & +k \int_{\Omega} c_{\varepsilon}\left|D^{2} \log c_{\varepsilon}\right|^{2}+k \int_{\Omega} \frac{\left|\nabla c_{\varepsilon}\right|^{4}}{c_{\varepsilon}^{3}} \\
& \leq C+K \int_{\Omega}\left|\nabla u_{\varepsilon}\right|^{2}-2 \int_{\Omega} \frac{\nabla c_{\varepsilon} \cdot \nabla n_{\varepsilon}}{1+\varepsilon n_{\varepsilon}} \quad \text { on }\left(0, T_{\max , \varepsilon}\right) .
\end{aligned}
$$

Lemma 2.6. For all $\eta>0$ there exists $C_{\eta}>0$ such that for all $\varepsilon>0$,

$$
\frac{d}{d t} \int_{\Omega}\left|u_{\varepsilon}\right|^{2}+\int_{\Omega}\left|\nabla u_{\varepsilon}\right|^{2} \leq \eta \int_{\Omega} n_{\varepsilon}^{2} \log n_{\varepsilon}+C_{\eta} \quad \text { on }\left(0, T_{\max , \varepsilon}\right) .
$$

Thanks to these lemmas, we can establish the estimate for $\frac{d \mathcal{F}_{\varepsilon}}{d t}$ which enables us to derive the desired estimate for $\mathcal{F}_{\varepsilon}$.

Lemma 2.7. There exist $C, k_{0}, K>0$ satisfying

$$
\begin{aligned}
& \frac{d}{d t}\left[\int_{\Omega} n_{\varepsilon} \log n_{\varepsilon}+\frac{\chi}{2} \int_{\Omega} \frac{\left|\nabla c_{\varepsilon}\right|^{2}}{c_{\varepsilon}}+K \chi \int_{\Omega}\left|u_{\varepsilon}\right|^{2}\right] \\
& +\frac{\mu}{4} \int_{\Omega} n_{\varepsilon}^{2} \log n_{\varepsilon}+\frac{4 m}{(m+1)^{2}} \int_{\Omega} \frac{\left|\nabla\left(n_{\varepsilon}+\varepsilon\right)^{\frac{m+1}{2}}\right|^{2}}{n_{\varepsilon}}+k_{0} \int_{\Omega} c_{\varepsilon}\left|D^{2} \log c_{\varepsilon}\right|^{2} \\
& +k_{0} \int_{\Omega} \frac{\left|\nabla c_{\varepsilon}\right|^{4}}{c_{\varepsilon}^{3}}+k_{0} \int_{\Omega}\left|\nabla u_{\varepsilon}\right|^{2} \leq C \quad \text { on }\left(0, T_{\max , \varepsilon}\right) \quad \text { for all } \varepsilon>0 .
\end{aligned}
$$


Proof. This lemma can be derived by a combination of Lemmas 2.4, 2.5 and 2.6 with $\eta:=\frac{\mu}{4 K \chi}$.

Now we are in a position to see the estimate for $\mathcal{F}_{\varepsilon}$ uniformly-in- $\varepsilon$.

Lemma 2.8. There exists $C>0$ such that

$$
\mathcal{F}_{\varepsilon}(t)=\int_{\Omega} n_{\varepsilon}(\cdot, t) \log n_{\varepsilon}(\cdot, t)+\frac{\chi}{2} \int_{\Omega} \frac{\left|\nabla c_{\varepsilon}(\cdot, t)\right|^{2}}{c_{\varepsilon}(\cdot, t)}+K \chi \int_{\Omega}\left|u_{\varepsilon}(\cdot, t)\right|^{2} \leq C
$$

for all $t \in\left(0, T_{\max , \varepsilon}\right)$ and all $\varepsilon>0$ and

$$
\begin{aligned}
& \int_{t}^{t+\tau} \int_{\Omega} n_{\varepsilon}^{2} \log n_{\varepsilon}+\int_{t}^{t+\tau} \int_{\Omega} \frac{\left|\nabla\left(n_{\varepsilon}+\varepsilon\right)^{\frac{m+1}{2}}\right|^{2}}{n_{\varepsilon}}+\int_{t}^{t+\tau} \int_{\Omega} c_{\varepsilon}\left|D^{2} \log c_{\varepsilon}\right|^{2} \leq C, \\
& \int_{t}^{t+\tau} \int_{\Omega} \frac{\left|\nabla c_{\varepsilon}\right|^{4}}{c_{\varepsilon}^{3}}+\int_{t}^{t+\tau} \int_{\Omega}\left|\nabla u_{\varepsilon}\right|^{2} \leq C, \\
& \int_{t}^{t+\tau} \int_{\Omega}\left|\nabla\left(n_{\varepsilon}+\varepsilon\right)^{\frac{m+1}{2}}\right|^{\frac{4}{3}}+\int_{\Omega}\left|\nabla c_{\varepsilon}\right|^{2}+\int_{t}^{t+\tau} \int_{\Omega}\left|\nabla c_{\varepsilon}\right|^{4}+\int_{t}^{t+\tau} \int_{\Omega} n_{\varepsilon}^{2} \leq C
\end{aligned}
$$

for all $t \in\left[0, T_{\max , \varepsilon}-\tau\right)$ and all $\varepsilon>0$, where $\tau:=\min \left\{1, \frac{1}{2} T_{\max , \varepsilon}\right\}$.

Proof. The proof is based on that of [14, Lemma 2.11]. Noticing from the inequalities $s \log s \leq \frac{1}{2 e}+s^{2} \log s, \int_{\Omega} \frac{\left|\nabla c_{\varepsilon}\right|^{2}}{c_{\varepsilon}} \leq\left\|c_{0}\right\|_{L^{\infty}(\Omega)} \int_{\Omega} \frac{\left|\nabla c_{\varepsilon}\right|^{4}}{c_{\varepsilon}^{3}}+|\Omega|$ (from Lemma 2.3) and $\int_{\Omega}\left|u_{\varepsilon}\right|^{2} \leq$ $C_{1} \int_{\Omega}\left|\nabla u_{\varepsilon}\right|^{2}$ with some $C_{1}>0$ (from the Poincaré inequality) that Lemma 2.7 implies

$$
\frac{d \mathcal{F}_{\varepsilon}}{d t}+k_{1} \mathcal{F}_{\varepsilon} \leq k_{2}
$$

with some $k_{1}, k_{2}>0$, we establish the boundedness of $\mathcal{F}_{\varepsilon}$ on $\left(0, T_{\max , \varepsilon}\right)$. Then for $\tau=$ $\min \left\{1, \frac{1}{2} T_{\max , \varepsilon}\right\}$ integrating the inequality $(2.3)$ over $(t, t+\tau)$ with Lemmas 2.2 and 2.3 implies this lemma.

Then we shall establish global existence in approximate problem (2.1) by using a Moser-Alikakos-type procedure.

Lemma 2.9. For all $\varepsilon \in(0,1), T_{\max , \varepsilon}=\infty$ holds.

Proof. The proof is based on that of [29, Lemma 3.9]. Assume that $T_{\max , \varepsilon}<\infty$ and put $p:=\min \{3+m, 4\}$. We shall first verify the $L^{p}$-estimate for $n_{\varepsilon}$. We see from the first equation and the fact $\nabla \cdot u_{\varepsilon}=0$ on $\Omega \times(0, \infty)$ that

$$
\begin{aligned}
\frac{1}{p} \frac{d}{d t} \int_{\Omega} n_{\varepsilon}^{p}= & \int_{\Omega} n_{\varepsilon}^{p-1} \nabla \cdot\left(m\left(n_{\varepsilon}+\varepsilon\right)^{m-1} \nabla n_{\varepsilon}-\chi \frac{n_{\varepsilon}}{1+\varepsilon n_{\varepsilon}} \nabla c_{\varepsilon}\right) \\
& +\int_{\Omega} n_{\varepsilon}^{p-1}\left(\kappa n_{\varepsilon}-\mu n_{\varepsilon}^{2}\right)-\frac{1}{p} \int_{\Omega} u_{\varepsilon} \cdot \nabla n_{\varepsilon}^{p} \\
= & -m(p-1) \int_{\Omega} n_{\varepsilon}^{p-2}\left(n_{\varepsilon}+\varepsilon\right)^{m-1}\left|\nabla n_{\varepsilon}\right|^{2} \\
& +\chi(p-1) \int_{\Omega} \frac{n_{\varepsilon}^{p-1}}{1+\varepsilon n_{\varepsilon}} \nabla n_{\varepsilon} \cdot \nabla c_{\varepsilon}+\kappa \int_{\Omega} n_{\varepsilon}^{p}-\mu \int_{\Omega} n_{\varepsilon}^{p+1} .
\end{aligned}
$$


Here, since $2 p-4+2(1-m)_{+}<p+1$, the Young inequality yields that

$$
\begin{aligned}
\chi(p-1) & \int_{\Omega} \frac{n_{\varepsilon}^{p-1}}{1+\varepsilon n_{\varepsilon}} \nabla n_{\varepsilon} \cdot \nabla c_{\varepsilon} \\
& \leq \frac{\chi(p-1)}{\varepsilon} \int_{\Omega} n_{\varepsilon}^{\frac{p-2}{2}}\left(n_{\varepsilon}+\varepsilon\right)^{\frac{m-1}{2}}\left|\nabla n_{\varepsilon}\right| n_{\varepsilon}^{\frac{p}{2}-1}\left(n_{\varepsilon}+\varepsilon\right)^{-\frac{m-1}{2}}\left|\nabla c_{\varepsilon}\right| \\
& \leq \frac{m(p-1)}{2} \int_{\Omega} n_{\varepsilon}^{p-2}\left(n_{\varepsilon}+\varepsilon\right)^{m-1}\left|\nabla n_{\varepsilon}\right|^{2}+\int_{\Omega} n_{\varepsilon}^{2 p-4}\left(n_{\varepsilon}+\varepsilon\right)^{2(1-m)}+C_{1} \int_{\Omega}\left|\nabla c_{\varepsilon}\right|^{4} \\
& \leq \frac{m(p-1)}{2} \int_{\Omega} n_{\varepsilon}^{p-2}\left(n_{\varepsilon}+\varepsilon\right)^{m-1}\left|\nabla n_{\varepsilon}\right|^{2}+\frac{\mu}{2} \int_{\Omega} n_{\varepsilon}^{p+1}+C_{2}+C_{1} \int_{\Omega}\left|\nabla c_{\varepsilon}\right|^{4}
\end{aligned}
$$

on $\left(0, T_{\max , \varepsilon}\right)$ with some $C_{1}=C_{1}(\varepsilon)>0$ and $C_{2}=C_{2}(\varepsilon)>0$, where we used the inequalities $(a+b)^{r} \leq 2^{r}\left(a^{r}+b^{r}\right)(a, b \geq 0, r>0)$ and $(a+b)^{r} \leq b^{r}(a, b \geq 0, r \leq 0)$ to obtain the last inequality. Therefore we obtain from the positivity of $n_{\varepsilon}$ that

$$
\frac{1}{p} \frac{d}{d t} \int_{\Omega} n_{\varepsilon}^{p} \leq C_{1} \int_{\Omega}\left|\nabla c_{\varepsilon}\right|^{4}+\kappa \int_{\Omega} n_{\varepsilon}^{p}+C_{2} .
$$

Thus it follows from Lemma 2.8 that

$$
\int_{\Omega} n_{\varepsilon}^{p} \leq C_{3} \quad \text { on }\left(0, T_{\max , \varepsilon}\right)
$$

where $C_{3}=C_{3}(\varepsilon)>0$. Then, aided by the $L^{2}$-estimate for $\nabla u_{\varepsilon}$ (from a testing argument), we can obtain that

$$
\left\|A^{\theta} u_{\varepsilon}(\cdot, t)\right\|_{L^{2}(\Omega)} \leq C_{4}
$$

for all $t \in\left(0, T_{\max , \varepsilon}\right)$ with some $C_{4}=C_{4}(\varepsilon)>0$. Then the continuous embedding $D\left(A^{\theta}\right) \hookrightarrow L^{\infty}(\Omega)$ implies the $L^{\infty}$-estimate for $u_{\varepsilon}$. By using these estimates a standard $L^{p}-L^{q}$ estimate for the Neumann heat semigroup on bounded domains and the inequality

$$
\|u(\cdot, t) \nabla c(\cdot, t)\|_{L^{3}(\Omega)} \leq\|u(\cdot, t)\|_{L^{\infty}(\Omega)}\|\nabla c(\cdot, t)\|_{L^{6}(\Omega)}^{\frac{1}{2}}\|\nabla c(\cdot, t)\|_{L^{2}(\Omega)}^{\frac{1}{2}}
$$

and the $L^{2}$-estimate for $\nabla c_{\varepsilon}$ from Lemma 2.8 imply the $L^{6}$-estimate for $\nabla c_{\varepsilon}$ (cf. an argument in the proof of $\left[\mathbf{2}\right.$, Lemma 3.10]). Finally we shall verify the $L^{\infty}$-estimate for $n_{\varepsilon}$. Put $\widetilde{n_{\varepsilon}}(x, t):=\max \left\{n_{\varepsilon}(x, t), s_{0}\right\}$ for $(x, t) \in \Omega \times\left(0, T_{\max , \varepsilon}\right)$ with some $s_{0}>0$. Then we can see from $\nabla \cdot u_{\varepsilon}=0$ in $\Omega \times\left(0, T_{\max , \varepsilon}\right)$ that

$$
\begin{aligned}
\frac{d}{d t} \int_{\Omega}{\widetilde{n_{\varepsilon}}}^{p} & +p(p-1) \int_{\Omega}\left(n_{\varepsilon}+\varepsilon\right)^{m-1}{\widetilde{n_{\varepsilon}}}^{p-2}\left|\nabla \widetilde{n_{\varepsilon}}\right|^{2} \\
& \leq p(p-1) \chi \int_{\Omega}{\widetilde{n_{\varepsilon}}}^{p-2} \frac{n_{\varepsilon}}{1+\varepsilon n_{\varepsilon}} \nabla c_{\varepsilon} \cdot \nabla \widetilde{n_{\varepsilon}}+\kappa \int_{\Omega}{\widetilde{n_{\varepsilon}}}^{p-1} n_{\varepsilon}
\end{aligned}
$$

on $\left(0, T_{\max , \varepsilon}\right)$. Thus, noting that

$$
\frac{n_{\varepsilon}}{1+\varepsilon n_{\varepsilon}} \nabla c_{\varepsilon} \in L^{\infty}\left(0, T_{\max , \varepsilon} ; L^{6}(\Omega)\right), \quad n_{\varepsilon} \in L^{\infty}\left(0, T_{\max , \varepsilon} ; L^{3+2 m}(\Omega)\right),
$$

from a Moser-Alikakos-type procedure (see the proof of [22, Lemma A.1]), we can attain that

$$
\left\|n_{\varepsilon}(\cdot, t)\right\|_{L^{\infty}(\Omega)} \leq C_{5}
$$

for all $t \in\left(0, T_{\max , \varepsilon}\right)$ with some $C_{5}=C_{5}(\varepsilon)>0$, which with extensibility criterion shows $T_{\max , \varepsilon}=\infty$ for each $\varepsilon \in(0,1)$. 


\section{Uniform-in- $\varepsilon$ estimates}

In this section we collect lemmas which are needed to show convergence of solutions of (2.1) as $\varepsilon \searrow 0$. Here the case that $m=1$ has already been dealt with in [14]. Thus we shall consider the case that $m>0$ with $m \neq 1$. From Lemma 2.8 we only know not some estimate for $\nabla n_{\varepsilon}$ but $L_{\text {loc }}^{\frac{4}{3}}\left([0, \infty) ; W^{1, \frac{4}{3}}(\Omega)\right)$-boundedness of $\left(\left(n_{\varepsilon}+\varepsilon\right)^{\frac{m+1}{2}}\right)_{\varepsilon \in(0,1)}$. However, it seems to be difficult to derive an enough regularity of $\partial_{t}\left(n_{\varepsilon}+\varepsilon\right)^{\frac{m+1}{2}}$ for each $m>0$. Therefore we need to establish an estimate for $\nabla\left(n_{\varepsilon}+\varepsilon\right)^{\gamma}$ with some $\gamma<\frac{m+1}{2}$. The following lemma is a cornerstone in the proof of Theorem 1.1.

Lemma 3.1. For all $T>0$ there exists a constant $C=C(T)>0$ such that

$$
\int_{0}^{T} \int_{\Omega}\left|\nabla\left(n_{\varepsilon}+\varepsilon\right)^{\frac{m}{2}}\right|^{2} \leq C \quad \text { on }(0, T) .
$$

Proof. In light of Lemma 2.8 we see that there exists $C_{1}=C_{1}(T)>0$ such that

$$
\begin{aligned}
\int_{0}^{T} \int_{\Omega}\left|\nabla\left(n_{\varepsilon}+\varepsilon\right)^{\frac{m}{2}}\right|^{2} & =\int_{0}^{T} \int_{\Omega} \frac{\left|\nabla\left(n_{\varepsilon}+\varepsilon\right)^{\frac{m+1}{2}}\right|^{2}}{n_{\varepsilon}+\varepsilon} \\
& \leq \int_{0}^{T} \int_{\Omega} \frac{\left|\nabla\left(n_{\varepsilon}+\varepsilon\right)^{\frac{m+1}{2}}\right|^{2}}{n_{\varepsilon}} \leq C_{1}
\end{aligned}
$$

with $C_{1}=C_{1}(T)>0$.

We next confirm the following lemma which will play an important role in deriving some time regularity of $\left(n_{\varepsilon}+\varepsilon\right)^{\frac{m}{2}}$.

Lemma 3.2. Let $m>0$ be such that $m \neq 1$. Then for all $T>0$ there exists a constant $C=C(T)>0$ such that for all $\varepsilon \in(0,1)$,

$$
\int_{\Omega}\left(n_{\varepsilon}+\varepsilon\right)^{m} \leq C
$$

on $(0, T)$ and

$$
\int_{0}^{T} \int_{\Omega}\left(n_{\varepsilon}+\varepsilon\right)^{2 m-3}\left|\nabla n_{\varepsilon}\right|^{2} \leq C
$$

hold.

Proof. From the first equation in (1.1) with $\nabla \cdot u_{\varepsilon}=0$ in $\Omega \times(0, \infty)$ we have

$$
\begin{aligned}
\frac{1}{m} \frac{d}{d t} \int_{\Omega}\left(n_{\varepsilon}+\varepsilon\right)^{m}= & -m(m-1) \int_{\Omega}\left(n_{\varepsilon}+\varepsilon\right)^{2 m-3}\left|\nabla n_{\varepsilon}\right|^{2} \\
& +(m-1) \chi \int_{\Omega}\left(n_{\varepsilon}+\varepsilon\right)^{m-2} \frac{n_{\varepsilon}}{1+\varepsilon n_{\varepsilon}} \nabla n_{\varepsilon} \cdot \nabla c_{\varepsilon} \\
& +\int_{\Omega}\left(n_{\varepsilon}+\varepsilon\right)^{m-1}\left(\kappa n_{\varepsilon}-\mu n_{\varepsilon}^{2}\right) .
\end{aligned}
$$


Here we note from the Young inequality that

$$
\begin{aligned}
\left|\chi(m-1) \int_{\Omega}\left(n_{\varepsilon}+\varepsilon\right)^{m-2} \frac{n_{\varepsilon}}{1+\varepsilon n_{\varepsilon}} \nabla n_{\varepsilon} \cdot \nabla c_{\varepsilon}\right| & \leq \chi|m-1| \int_{\Omega}\left(n_{\varepsilon}+\varepsilon\right)^{m-1}\left|\nabla n_{\varepsilon}\right|\left|\nabla c_{\varepsilon}\right| \\
\leq & \frac{m|m-1|}{2} \int_{\Omega}\left(n_{\varepsilon}+\varepsilon\right)^{2 m-3}\left|\nabla n_{\varepsilon}\right|^{2} \\
& +\int_{\Omega}\left(n_{\varepsilon}+\varepsilon\right)^{2}+C_{1} \int_{\Omega}\left|\nabla c_{\varepsilon}\right|^{4},
\end{aligned}
$$

where $C_{1}>0$. In the case that $m>1$, since $m-1>0$, we obtain that

$$
\begin{aligned}
\frac{1}{m} \frac{d}{d t} \int_{\Omega}\left(n_{\varepsilon}+\varepsilon\right)^{m}+ & \frac{m(m-1)}{2} \int_{\Omega}\left(n_{\varepsilon}+\varepsilon\right)^{2 m-3}\left|\nabla n_{\varepsilon}\right|^{2} \\
& \leq \int_{\Omega}\left(n_{\varepsilon}+\varepsilon\right)^{2}+C_{1} \int_{\Omega}\left|\nabla c_{\varepsilon}\right|^{4}+\kappa \int_{\Omega}\left(n_{\varepsilon}+\varepsilon\right)^{m}
\end{aligned}
$$

which together with Lemma 2.8 shows that there is $C_{2}=C_{2}(T)>0$ such that

$$
\int_{\Omega}\left(n_{\varepsilon}+\varepsilon\right)^{m} \leq C_{2}
$$

on $(0, T)$ for all $\varepsilon \in(0,1)$ and

$$
\int_{0}^{T} \int_{\Omega}\left(n_{\varepsilon}+\varepsilon\right)^{2 m-3}\left|\nabla n_{\varepsilon}\right|^{2} \leq C_{2}
$$

for all $\varepsilon \in(0,1)$. On the other hand, in the case that $0<m<1$, we have from (3.1) that

$$
\begin{aligned}
\frac{1}{m} \frac{d}{d t} \int_{\Omega}\left(n_{\varepsilon}+\varepsilon\right)^{m} \geq & \frac{m(1-m)}{2} \int_{\Omega}\left(n_{\varepsilon}+\varepsilon\right)^{2 m-3}\left|\nabla n_{\varepsilon}\right|^{2} \\
& -\int_{\Omega}\left(n_{\varepsilon}+\varepsilon\right)^{2}-C_{1} \int_{\Omega}\left|\nabla c_{\varepsilon}\right|^{4}-\mu \int_{\Omega}\left(n_{\varepsilon}+\varepsilon\right)^{m+1} .
\end{aligned}
$$

Thus, integrating it over $(0, T)$, we derive from applications of the Young inequality

$$
\int_{\Omega}\left(n_{\varepsilon}+\varepsilon\right)^{m} \leq m \int_{\Omega}\left(n_{\varepsilon}+1\right)+(1-m)|\Omega|
$$

and

$$
\int_{0}^{T} \int_{\Omega}\left(n_{\varepsilon}+\varepsilon\right)^{m+1} \leq \frac{m+1}{2} \int_{0}^{T} \int_{\Omega}\left(n_{\varepsilon}+1\right)^{2}+\frac{1-m}{2}|\Omega| T
$$

with Lemma 2.2 that

$$
\frac{m(1-m)}{2} \int_{0}^{T} \int_{\Omega}\left(n_{\varepsilon}+\varepsilon\right)^{2 m-3}\left|\nabla n_{\varepsilon}\right|^{2} \leq C_{3}
$$

with some $C_{3}=C_{3}(T)>0$. 
In order to see some time regularity of $\left(n_{\varepsilon}+\varepsilon\right)^{\frac{m}{2}}$ we will give the following lemma.

Lemma 3.3. Let $m>0$ be such that $m \neq 1$. Then for all $T>0$ there exists a constant $C=C(T)>0$ such that for all $\varepsilon \in(0,1)$,

$$
\int_{\Omega}\left(n_{\varepsilon}+\varepsilon\right)^{m-1} \leq C
$$

on $(0, T)$ and

$$
\int_{0}^{T} \int_{\Omega}\left(n_{\varepsilon}+\varepsilon\right)^{2 m-4}\left|\nabla n_{\varepsilon}\right|^{2} \leq C
$$

hold.

Proof. We derive from the first equation in (2.1) and integration by parts that

$$
\begin{aligned}
\frac{d}{d t} \int_{\Omega}\left(n_{\varepsilon}+\varepsilon\right)^{m-1}= & -m(m-1)(m-2) \int_{\Omega}\left(n_{\varepsilon}+\varepsilon\right)^{2 m-4}\left|\nabla n_{\varepsilon}\right|^{2} \\
& +\chi(m-1)(m-2) \int_{\Omega}\left(n_{\varepsilon}+\varepsilon\right)^{m-3} \frac{n_{\varepsilon}}{1+\varepsilon n_{\varepsilon}} \nabla n_{\varepsilon} \cdot \nabla c_{\varepsilon} \\
& +\kappa(m-1) \int_{\Omega}\left(n_{\varepsilon}+\varepsilon\right)^{m-2} n_{\varepsilon}-\mu(m-1) \int_{\Omega}\left(n_{\varepsilon}+\varepsilon\right)^{m-2} n_{\varepsilon}^{2} .
\end{aligned}
$$

Now we note from the Young inequality and Lemma 2.8 that

$$
\begin{aligned}
& \left|\chi(m-1)(m-2) \int_{\Omega}\left(n_{\varepsilon}+\varepsilon\right)^{m-3} \frac{n_{\varepsilon}}{1+\varepsilon n_{\varepsilon}} \nabla n_{\varepsilon} \cdot \nabla c_{\varepsilon}\right| \\
& \leq \frac{m|(m-1)(m-2)|}{2} \int_{\Omega}\left(n_{\varepsilon}+\varepsilon\right)^{2 m-4}\left|\nabla n_{\varepsilon}\right|^{2}+C_{1} \int_{\Omega}\left|\nabla c_{\varepsilon}\right|^{2} \\
& \leq \frac{m|(m-1)(m-2)|}{2} \int_{\Omega}\left(n_{\varepsilon}+\varepsilon\right)^{2 m-4}\left|\nabla n_{\varepsilon}\right|^{2}+C_{2}
\end{aligned}
$$

with $C_{1}, C_{2}>0$. We first consider the cases that $m>2$ and $0<m<1$. Since it follows that $(m-1)(m-2)>0$ and that if $m>2$ then

$$
\kappa(m-1) \int_{\Omega}\left(n_{\varepsilon}+\varepsilon\right)^{m-2} n_{\varepsilon}-\mu(m-1) \int_{\Omega}\left(n_{\varepsilon}+\varepsilon\right)^{m-2} n_{\varepsilon}^{2} \leq \kappa(m-1) \int_{\Omega}\left(n_{\varepsilon}+\varepsilon\right)^{m-1}
$$

and if $0<m<1$ then

$$
\kappa(m-1) \int_{\Omega}\left(n_{\varepsilon}+\varepsilon\right)^{m-2} n_{\varepsilon}-\mu(m-1) \int_{\Omega}\left(n_{\varepsilon}+\varepsilon\right)^{m-2} n_{\varepsilon}^{2} \leq \mu(1-m) \int_{\Omega}\left(n_{\varepsilon}+\varepsilon\right)^{m} \leq C_{3}
$$

with some $C_{3}=C_{3}(T)>0$ (from Lemma 3.2), we infer from (3.2) that

$$
\begin{aligned}
& \frac{d}{d t} \int_{\Omega}\left(n_{\varepsilon}+\varepsilon\right)^{m-1}+\frac{m(m-1)(m-2)}{2} \int_{\Omega}\left(n_{\varepsilon}+\varepsilon\right)^{2 m-4}\left|\nabla n_{\varepsilon}\right|^{2} \\
& \leq \kappa|m-1| \int_{\Omega}\left(n_{\varepsilon}+\varepsilon\right)^{m-1}+C_{4}
\end{aligned}
$$


with some $C_{4}=C_{4}(T)>0$, and hence there exists a constant $C_{5}=C_{5}(T)>0$ such that for all $\varepsilon \in(0,1)$,

$$
\int_{\Omega}\left(n_{\varepsilon}+\varepsilon\right)^{m-1} \leq C_{5}
$$

on $(0, T)$ and

$$
\int_{\Omega}\left(n_{\varepsilon}+\varepsilon\right)^{2 m-4}\left|\nabla n_{\varepsilon}\right|^{2} \leq C_{5}
$$

On the other hand, in the case that $1<m<2$, we see from (3.2) and (3.3) that

$$
\begin{aligned}
\frac{d}{d t} \int_{\Omega}\left(n_{\varepsilon}+\varepsilon\right)^{m-1} \geq & \frac{m(m-1)(2-m)}{2} \int_{\Omega}\left(n_{\varepsilon}+\varepsilon\right)^{2 m-4}\left|\nabla n_{\varepsilon}\right|^{2}-C_{6} \\
& -\mu(m-1) \int_{\Omega}\left(n_{\varepsilon}+\varepsilon\right)^{m}
\end{aligned}
$$

with some $C_{6}>0$. Hence, noticing that $\int_{\Omega}\left(n_{\varepsilon}+\varepsilon\right)^{m-1} \leq(m-1) \int_{\Omega}\left(n_{\varepsilon}+1\right)+(2-m)|\Omega|$ and $(m-1)(2-m)>0$, we derive from Lemmas 2.2 and 3.2 that

$$
\frac{m(m-1)(2-m)}{2} \int_{0}^{T} \int_{\Omega}\left(n_{\varepsilon}+\varepsilon\right)^{2 m-4}\left|\nabla n_{\varepsilon}\right|^{2} \leq C_{7}
$$

with some $C_{7}=C_{7}(T)>0$. Finally, in the case that $m=2$, Lemma 3.1 implies this lemma.

The following time regularity of $\left(n_{\varepsilon}+\varepsilon\right)^{\frac{m}{2}}$ will be useful in applying a Lions-Aubin type lemma later.

Lemma 3.4. Let $m>0$ be such that $m \neq 1$. Then for all $T>0$ there exists a constant $C=C(T)>0$ satisfying

$$
\left\|\partial_{t}\left(n_{\varepsilon}+\varepsilon\right)^{\frac{m}{2}}\right\|_{L^{1}\left(0, T ;\left(W_{0}^{2,4}(\Omega)\right)^{*}\right)} \leq C \quad \text { for all } \varepsilon \in(0,1) .
$$

Proof. Let $T>0$ and let $\psi \in L^{\infty}\left(0, T ; W_{0}^{2,4}(\Omega)\right)$. The first equation in (2.1) and integration by parts yield that

$$
\begin{aligned}
\int_{0}^{T} \int_{\Omega}\left(\partial_{t}\left(n_{\varepsilon}+\varepsilon\right)^{\frac{m}{2}}\right) \psi= & -\int_{0}^{T} \int_{\Omega} \psi u_{\varepsilon} \cdot \nabla\left(n_{\varepsilon}+\varepsilon\right)^{\frac{m}{2}} \\
& -\frac{m^{2}}{m+1}\left(\frac{m}{2}-1\right) \int_{0}^{T} \int_{\Omega} \psi \frac{\nabla\left(n_{\varepsilon}+\varepsilon\right)^{\frac{m+1}{2}}}{\left(n_{\varepsilon}+\varepsilon\right)^{\frac{1}{2}}} \cdot\left(n_{\varepsilon}+\varepsilon\right)^{m-2} \nabla n_{\varepsilon} \\
& -\frac{m^{2}}{2} \int_{0}^{T} \int_{\Omega}\left(n_{\varepsilon}+\varepsilon\right)^{m-\frac{3}{2}} \nabla n_{\varepsilon} \cdot\left(n_{\varepsilon}+\varepsilon\right)^{\frac{m-1}{2}} \nabla \psi \\
& +\frac{m \chi}{m+1}\left(\frac{m}{2}-1\right) \int_{0}^{T} \int_{\Omega} \frac{n_{\varepsilon} \psi}{\left(1+\varepsilon n_{\varepsilon}\right)\left(n_{\varepsilon}+\varepsilon\right)} \frac{\nabla\left(n_{\varepsilon}+\varepsilon\right)^{\frac{m+1}{2}}}{\left(n_{\varepsilon}+\varepsilon\right)^{\frac{1}{2}}} \cdot \nabla c_{\varepsilon} \\
& +\frac{m \chi}{2} \int_{0}^{T} \int_{\Omega}\left(n_{\varepsilon}+\varepsilon\right)^{\frac{m}{2}} \frac{n_{\varepsilon}}{\left(1+\varepsilon n_{\varepsilon}\right)\left(n_{\varepsilon}+\varepsilon\right)} \nabla c_{\varepsilon} \cdot \nabla \psi \\
& +\frac{m \kappa}{2} \int_{0}^{T} \int_{\Omega}\left(n_{\varepsilon}+\varepsilon\right)^{\frac{m}{2}-1} n_{\varepsilon} \psi-\frac{m \mu}{2} \int_{0}^{T} \int_{\Omega}\left(n_{\varepsilon}+\varepsilon\right)^{\frac{m}{2}-1} n_{\varepsilon}^{2} \psi .
\end{aligned}
$$


Then, noting from the Young inequality that

$$
\begin{gathered}
\left|\int_{0}^{T} \int_{\Omega} \psi u_{\varepsilon} \cdot \nabla\left(n_{\varepsilon}+\varepsilon\right)^{\frac{m}{2}}\right| \leq \frac{\|\psi\|_{L^{\infty}(\Omega \times(0, T))}}{2}\left(\int_{0}^{T} \int_{\Omega}\left|u_{\varepsilon}\right|^{2}+\int_{0}^{T} \int_{\Omega}\left|\nabla\left(n_{\varepsilon}+\varepsilon\right)^{\frac{m}{2}}\right|^{2}\right), \\
\left|\int_{0}^{T} \int_{\Omega} \psi \frac{\nabla\left(n_{\varepsilon}+\varepsilon\right)^{\frac{m+1}{2}}}{\left(n_{\varepsilon}+\varepsilon\right)^{\frac{1}{2}}} \cdot\left(n_{\varepsilon}+\varepsilon\right)^{m-2} \nabla n_{\varepsilon}\right| \\
\leq \frac{\|\psi\|_{L^{\infty}(\Omega \times(0, T))}}{2} \int_{0}^{T} \int_{\Omega}\left(\frac{\left|\nabla\left(n_{\varepsilon}+\varepsilon\right)^{\frac{m+1}{2}}\right|^{2}}{n_{\varepsilon}}+\int_{0}^{T} \int_{\Omega}\left(n_{\varepsilon}+\varepsilon\right)^{2 m-4}\left|\nabla n_{\varepsilon}\right|^{2}\right)
\end{gathered}
$$

and

$$
\begin{aligned}
& \left|\int_{0}^{T} \int_{\Omega}\left(n_{\varepsilon}+\varepsilon\right)^{m-\frac{3}{2}} \nabla n_{\varepsilon} \cdot\left(n_{\varepsilon}+\varepsilon\right)^{\frac{m-1}{2}} \nabla \psi\right| \\
& \quad \leq \frac{\|\nabla \psi\|_{L^{\infty}(\Omega \times(0, T))}}{2}\left(\int_{0}^{T} \int_{\Omega}\left(n_{\varepsilon}+\varepsilon\right)^{2 m-3}\left|\nabla n_{\varepsilon}\right|^{2}+\int_{0}^{T} \int_{\Omega}\left(n_{\varepsilon}+\varepsilon\right)^{m-1}\right), \\
& \left|\int_{0}^{T} \int_{\Omega} \psi \frac{n_{\varepsilon}}{\left(1+\varepsilon n_{\varepsilon}\right)\left(n_{\varepsilon}+\varepsilon\right)} \frac{\nabla\left(n_{\varepsilon}+\varepsilon\right)^{\frac{m+1}{2}}}{\left(n_{\varepsilon}+\varepsilon\right)^{\frac{1}{2}}} \cdot \nabla c_{\varepsilon}\right| \\
& \leq \frac{\|\psi\|_{L^{\infty}(\Omega \times(0, T))}}{2}\left(\int_{0}^{T} \int_{\Omega} \frac{\left|\nabla\left(n_{\varepsilon}+\varepsilon\right)^{\frac{m+1}{2}}\right|^{2}}{n_{\varepsilon}}+\int_{0}^{T} \int_{\Omega}\left|\nabla c_{\varepsilon}\right|^{2}\right)
\end{aligned}
$$

as well as

$$
\begin{aligned}
& \left|\int_{0}^{T} \int_{\Omega}\left(n_{\varepsilon}+\varepsilon\right)^{\frac{m}{2}} \frac{n_{\varepsilon}}{\left(1+\varepsilon n_{\varepsilon}\right)\left(n_{\varepsilon}+\varepsilon\right)} \nabla c_{\varepsilon} \cdot \nabla \psi\right| \\
& \quad \leq \frac{\|\nabla \psi\|_{L^{\infty}(\Omega \times(0, T))}}{2}\left(\int_{0}^{T} \int_{\Omega}\left(n_{\varepsilon}+\varepsilon\right)^{m}+\int_{0}^{T} \int_{\Omega}\left|\nabla c_{\varepsilon}\right|^{2}\right)
\end{aligned}
$$

with

$$
\begin{array}{r}
\left|\frac{m \kappa}{2} \int_{0}^{T} \int_{\Omega}\left(n_{\varepsilon}+\varepsilon\right)^{\frac{m}{2}-1} n_{\varepsilon} \psi-\frac{m \mu}{2} \int_{0}^{T} \int_{\Omega}\left(n_{\varepsilon}+\varepsilon\right)^{\frac{m}{2}-1} n_{\varepsilon}^{2} \psi\right| \\
\leq\|\psi\|_{L^{\infty}(\Omega \times(0, T))}\left(\int_{0}^{T} \int_{\Omega}\left(n_{\varepsilon}+\varepsilon\right)^{\max \{m, 2\}}+C_{1} T\right)
\end{array}
$$

for some $C_{1}>0$ (from the fact that $\frac{m}{2}+1 \leq \max \{m, 2\}$ ), we obtain from Lemmas 2.8, $3.1,3.2$ and 3.3 together with the continuous embedding $W_{0}^{2,4}(\Omega) \hookrightarrow W^{1, \infty}(\Omega)$ that

$$
\int_{0}^{T} \int_{\Omega}\left(\partial_{t}\left(n_{\varepsilon}+\varepsilon\right)^{\frac{m}{2}}\right) \psi \leq C_{2}\|\psi\|_{L^{\infty}\left(0, T ; W^{2,4}(\Omega)\right)}
$$

with some $C_{2}=C_{2}(T)>0$. Therefore a standard duality argument enables us to see this lemma.

We also give the following lemma concerned with time regularities of $c_{\varepsilon}$ and $u_{\varepsilon}$. 
Lemma 3.5. For all $T>0$ there exists $C=C(T)>0$ satisfying

$\left\|\left(c_{\varepsilon}\right)_{t}\right\|_{L^{2}\left(0, T ;\left(W_{0}^{1,2}(\Omega)\right)^{*}\right)} \leq C \quad$ and $\quad\left\|\left(u_{\varepsilon}\right)_{t}\right\|_{L^{2}\left(0, T ;\left(W_{0}^{1,3}(\Omega)\right)^{*}\right)} \leq C \quad$ for all $\varepsilon \in(0,1)$.

Proof. This lemma can be proved from the same arguments as those in the proofs of $[\mathbf{1 4}$, Lemmas 2.14 and 2.15].

Finally we give an estimate for $\nabla\left(n_{\varepsilon}+\varepsilon\right)^{m}$ to see convergence of $\int_{0}^{T} \int_{\Omega} \nabla\left(n_{\varepsilon}+\varepsilon\right)^{m} \cdot \nabla \varphi$ for all $\varphi \in C_{0}^{\infty}(\bar{\Omega} \times[0, \infty))$.

Lemma 3.6. Let $m>0$ be such that $m \neq 1$. Then for all $T>0$ and all $r \in\left(1, \frac{4}{3}\right]$ there exists a constant $C=C(T)>0$ such that

$$
\left\|\nabla\left(n_{\varepsilon}+\varepsilon\right)^{m}\right\|_{L^{r}\left(0, T ; L^{r}(\Omega)\right)} \leq C \quad \text { for all } \varepsilon \in(0,1) .
$$

Proof. Let $r=\frac{4}{3}$. Since $\frac{r}{2-r}=2$, the Young inequality yields

$$
\begin{aligned}
\int_{0}^{T} \int_{\Omega}\left|\nabla\left(n_{\varepsilon}+\varepsilon\right)^{m}\right|^{r} & =\int_{0}^{T} \int_{\Omega}\left(n_{\varepsilon}+\varepsilon\right)^{(m-1) r}\left|\nabla n_{\varepsilon}\right|^{r} \\
& \leq \int_{0}^{T} \int_{\Omega}\left(n_{\varepsilon}+\varepsilon\right)^{\frac{r}{2-r}}+C_{1} \int_{0}^{T} \int_{\Omega}\left(n_{\varepsilon}+\varepsilon\right)^{2 m-3}\left|\nabla n_{\varepsilon}\right|^{2} \\
& \leq \int_{0}^{T} \int_{\Omega}\left(n_{\varepsilon}+1\right)^{2}+C_{1} \int_{0}^{T} \int_{\Omega}\left(n_{\varepsilon}+\varepsilon\right)^{2 m-3}\left|\nabla n_{\varepsilon}\right|^{2}
\end{aligned}
$$

with some $C_{1}>0$. Therefore Lemmas 2.8 and 3.2 lead to this lemma.

\section{Convergence: Proof of Theorem 1.1}

In this section we consider convergence of solutions of approximate problem (2.1) and then prove Theorem 1.1. We first state the following result which can be obtained from the previous estimates in Section 3.

Lemma 4.1. There exist a sequence $\left(\varepsilon_{j}\right)_{j \in \mathbb{N}}$ such that $\varepsilon_{j} \searrow 0$ as $j \rightarrow \infty$ and functions $n, c, u$ such that

$$
\begin{aligned}
& n \in L_{\mathrm{loc}}^{2}\left([0, \infty) ; L^{2}(\Omega)\right), \\
& c \in L_{\mathrm{loc}}^{2}\left([0, \infty) ; W^{1,2}(\Omega)\right), \\
& u \in L_{\mathrm{loc}}^{2}\left([0, \infty) ; W_{0, \sigma}^{1,2}(\Omega)\right)
\end{aligned}
$$

and that for all $p \in[1,6)$,

$$
\begin{aligned}
\left(n_{\varepsilon}+\varepsilon\right)^{\frac{m}{2}} & \rightarrow n^{\frac{m}{2}} & & \text { in } L_{\mathrm{loc}}^{2}\left([0, \infty) ; L^{p}(\Omega)\right) \text { and a.e. in } \Omega \times(0, \infty), \\
n_{\varepsilon} & \rightarrow n & & \text { in } L_{\mathrm{loc}}^{2}\left([0, \infty) ; L^{2}(\Omega)\right), \\
c_{\varepsilon} & \rightarrow c & & \text { in } C_{\mathrm{loc}}^{0}\left([0, \infty) ; L^{p}(\Omega)\right), \\
u_{\varepsilon} & \rightarrow u & & \text { in } L_{\mathrm{loc}}^{2}\left([0, \infty) ; L^{p}(\Omega)\right), \\
\nabla c_{\varepsilon} & \rightarrow \nabla c & & \text { weakly in } L_{\mathrm{loc}}^{4}\left([0, \infty) ; L^{4}(\Omega)\right), \\
\nabla u_{\varepsilon} & \rightarrow \nabla u & & \text { weakly in } L_{\mathrm{loc}}^{2}\left([0, \infty) ; L^{2}(\Omega)\right), \\
Y_{\varepsilon} u_{\varepsilon} & \rightarrow u & & \text { in } L_{\mathrm{loc}}^{2}\left([0, \infty) ; L^{2}(\Omega)\right)
\end{aligned}
$$

as $\varepsilon=\varepsilon_{j} \searrow 0$. 
Proof. Let $T>0$. Thanks to Lemmas 3.1, 3.2 and 3.4, we have that

$$
\left(\left(n_{\varepsilon}+\varepsilon\right)^{\frac{m}{2}}\right)_{\varepsilon \in(0,1)} \text { is bounded in } L^{2}\left(0, T ; W^{1,2}(\Omega)\right)
$$

and

$$
\left(\partial_{t}\left(n_{\varepsilon}+\varepsilon\right)^{\frac{m}{2}}\right)_{\varepsilon \in(0,1)} \text { is bounded in } L^{1}\left(0, T ;\left(W_{0}^{2,4}(\Omega)^{*}\right) .\right.
$$

Therefore, aided by the compact embedding $W^{1,2}(\Omega) \hookrightarrow L^{p}(\Omega)$ for all $p \in[1,6)$ and the continuous embedding $L^{p}(\Omega) \hookrightarrow\left(W_{0}^{2,4}(\Omega)\right)^{*}$, we can see from a Lions-Aubin type lemma (see [20, Corollary 4]) that $\left(\left(n_{\varepsilon}+\varepsilon\right)^{\frac{m}{2}}\right)_{\varepsilon \in(0,1)}$ is relatively compact in $L^{2}\left(0, T ; L^{p}(\Omega)\right)$, which means that there are a sequence $\left(\varepsilon_{j}\right)_{j \in \mathbb{N}} \searrow 0$ and a function $v \in L^{2}\left(0, T ; L^{p}(\Omega)\right)$ such that $\left(n_{\varepsilon}+\varepsilon\right)^{\frac{m}{2}} \rightarrow v$ in $L^{2}\left(0, T ; L^{p}(\Omega)\right)$ as $\varepsilon=\varepsilon_{j} \searrow 0$. Then by putting $n:=v^{\frac{2}{m}}$ we have (4.1), which yields that $n_{\varepsilon} \rightarrow n$ a.e. in $\Omega \times(0, \infty)$ as $\varepsilon=\varepsilon_{j} \searrow 0$. The rest of the proof is mainly based on arguments in the proof of [14, Proposition 2.1]; thus we will give a short proof. Since a uniform bound on $\int_{0}^{T} \int_{\Omega} \Phi\left(n_{\varepsilon}^{2}\right)$, where $\Phi(s):=\frac{s}{2} \log (s)$ for $s>0$, derives from the Dunford-Pettis theorem (cf. [4, Lemma IV 8.9]) that $\left(n_{\varepsilon}^{2}\right)_{\varepsilon \in(0,1)}$ is weakly relatively precompact in $L^{1}(\Omega \times(0, T))$, we obtain that there is a subsequence of $\left(\varepsilon_{j}\right)_{j \in \mathbb{N}}$ such that $\int_{0}^{T} \int_{\Omega} n_{\varepsilon}^{2} \rightarrow \int_{0}^{T} \int_{\Omega} n^{2}$ as $\varepsilon=\varepsilon_{j} \searrow 0$. This together with the convergence $n_{\varepsilon} \rightarrow n$ weakly in $L^{2}\left(0, T ; L^{2}(\Omega)\right.$ ) as $\varepsilon=\varepsilon_{j} \searrow 0$ (from Lemma 2.8) yields that (4.2) along a further subsequence. On the other hand, by virtue of Lemmas 2.3, 2.8 and 3.5, we can establish that $\left(c_{\varepsilon}\right)_{\varepsilon \in(0,1)}$ and $\left(\left(c_{\varepsilon}\right)_{t}\right)_{\varepsilon \in(0,1)}$ are bounded in $L^{\infty}\left(0, T ; W^{1,2}(\Omega)\right)$ and in $L^{2}\left(0, T ;\left(W_{0}^{1,2}(\Omega)\right)^{*}\right)$, respectively, as well as $\left(u_{\varepsilon}\right)_{\varepsilon \in(0,1)}$ and $\left(\left(u_{\varepsilon}\right)_{t}\right)_{\varepsilon \in(0,1)}$ are bounded in $L^{2}\left(0, T ; W_{\sigma}^{1,2}\right)$ and in $L^{2}\left(0, T ;\left(W_{\sigma}^{1,3}(\Omega)\right)^{*}\right)$, respectively. Thus [20, Corollary 4] again implies (4.3) and (4.4), and then Lemma 2.8 leads to the convergences (4.5) and (4.6) along a further subsequence. Finally, noticing that $\left\|Y_{\varepsilon} u_{\varepsilon}(\cdot, t)-u(\cdot, t)\right\|_{L^{2}(\Omega)} \rightarrow 0$ as $\varepsilon=\varepsilon_{j} \searrow 0$ a.e. $t>0$ and $\left\|Y_{\varepsilon} u_{\varepsilon}(\cdot, t)-u(\cdot, t)\right\|_{L^{2}(\Omega)}^{2} \leq C$ for all $t>0$ and all $\varepsilon>0$ in view of Lemma 2.8, we can establish from the dominated convergence theorem that (4.7) along a further subsequence.

We then provide convergence of $\nabla\left(n_{\varepsilon}+\varepsilon\right)^{m}$ from Lemma 3.6.

Lemma 4.2. Let $m>0$ be such that $m \neq 1$. Then the function $n$ obtained in Lemma 4.1 satisfies that $n^{m} \in L_{\mathrm{loc}}^{\frac{4}{3}}\left([0, \infty) ; W^{1, \frac{4}{3}}(\Omega)\right)$ and

$$
\nabla\left(n_{\varepsilon}+\varepsilon\right)^{m} \rightarrow \nabla n^{m} \quad \text { weakly in } L_{\mathrm{loc}}^{\frac{4}{3}}\left([0, \infty) ; L^{\frac{4}{3}}(\Omega)\right)
$$

as $\varepsilon=\varepsilon_{j} \searrow 0$.

Proof. Let $T>0$. Since the Poincaré-Wirtinger inequality yields that

$$
\int_{0}^{T}\left\|\left(n_{\varepsilon}+\varepsilon\right)^{m}\right\|_{L^{\frac{4}{3}(\Omega)}}^{\frac{4}{3}} \leq C_{1} \int_{0}^{T}\left\|\nabla\left(n_{\varepsilon}+\varepsilon\right)^{m}\right\|_{L^{\frac{4}{3}(\Omega)}}^{\frac{4}{3}}+C_{1} \int_{0}^{T}|\Omega|^{-\frac{1}{4}}\left\|\left(n_{\varepsilon}+\varepsilon\right)^{m}\right\|_{L^{1}(\Omega)}
$$

with some $C_{1}>0$, we first note from the Fatou lemma and Lemmas 3.2, 3.6 that

$$
\int_{0}^{T}\left\|n^{m}\right\|_{L^{\frac{4}{3}(\Omega)}}^{\frac{4}{3}} \leq \liminf _{\varepsilon \searrow 0} \int_{0}^{T}\left\|\left(n_{\varepsilon}+\varepsilon\right)^{m}\right\|_{L^{\frac{4}{3}(\Omega)}}^{\frac{4}{3}} \leq C_{2}
$$


with some $C_{2}=C_{2}(T)>0$, which implies that $n^{m} \in L^{\frac{4}{3}}\left(0, T ; L^{\frac{4}{3}}(\Omega)\right)$. We next have from Lemma 3.6 that there exist a subsequence of $\left(\varepsilon_{j}\right)_{j \in \mathbb{N}}$ obtained in Lemma 4.1 (again denoted by $\left.\left(\varepsilon_{j}\right)_{j \in \mathbb{N}}\right)$ and a function $w \in L^{\frac{4}{3}}\left(0, T ; L^{\frac{4}{3}}(\Omega)\right)$ such that

$$
\nabla\left(n_{\varepsilon}+\varepsilon\right)^{m} \rightarrow w \quad \text { weakly in } L^{\frac{4}{3}}\left(0, T ; L^{\frac{4}{3}}(\Omega)\right)
$$

as $\varepsilon=\varepsilon_{j} \searrow 0$. In order to verify that $w=\nabla n^{m}$, it is enough to confirm that $\left(n_{\varepsilon}+\varepsilon\right)^{m} \rightarrow$ $n^{m}$ in $L^{1}\left(0, T ; L^{1}(\Omega)\right)$ as $\varepsilon=\varepsilon_{j} \searrow 0$. Now, since we have already known that $\left(n_{\varepsilon}+\varepsilon\right)^{m}$ is uniform integrable (from Lemma 3.2) and $\left(n_{\varepsilon}+\varepsilon\right)^{m} \rightarrow n^{m}$ a.e. in $\Omega \times(0, \infty)$ as $\varepsilon=\varepsilon_{j} \searrow 0$, the Vitali convergence theorem entails that

$$
\left(n_{\varepsilon}+\varepsilon\right)^{m} \rightarrow n^{m} \quad \text { in } L^{1}\left(0, T ; L^{1}(\Omega)\right)
$$

as $\varepsilon=\varepsilon_{j} \searrow 0$. Thanks to this strong convergence, we can verify that $w=\nabla n^{m}$, which together with $w \in L^{\frac{4}{3}}\left(0, T ; L^{\frac{4}{3}}(\Omega)\right)$ shows that $n^{m} \in L^{\frac{4}{3}}\left(0, T ; W^{1, \frac{4}{3}}(\Omega)\right)$.

We will establish global existence of weak solutions to (1.1) from convergences obtained in Lemmas 4.1 and 4.2 .

Proof of Theorem 1.1. Let $\varphi \in C_{0}^{\infty}(\bar{\Omega} \times[0, \infty))$ and $\psi \in C_{0, \sigma}^{\infty}(\Omega \times[0, \infty))$. Testing each equations in (2.1) by these functions and using integration by parts, we can see that

$$
\begin{aligned}
& -\int_{0}^{\infty} \int_{\Omega} n_{\varepsilon} \varphi_{t}-\int_{\Omega} n_{0} \varphi(\cdot, 0)-\int_{0}^{\infty} \int_{\Omega} n_{\varepsilon} u_{\varepsilon} \cdot \nabla \varphi \\
& =-\int_{0}^{\infty} \int_{\Omega} \nabla\left(n_{\varepsilon}+\varepsilon\right)^{m} \cdot \nabla \varphi+\chi \int_{0}^{\infty} \int_{\Omega} \frac{n_{\varepsilon}}{1+\varepsilon n_{\varepsilon}} \nabla c_{\varepsilon} \cdot \nabla \varphi+\int_{0}^{\infty} \int_{\Omega}\left(\kappa n_{\varepsilon}-\mu n_{\varepsilon}^{2}\right) \varphi \\
& -\int_{0}^{\infty} \int_{\Omega} c_{\varepsilon} \varphi_{t}-\int_{\Omega} c_{0} \varphi(\cdot, 0)-\int_{0}^{\infty} \int_{\Omega} c_{\varepsilon} u_{\varepsilon} \cdot \nabla \varphi \\
& =-\int_{0}^{\infty} \int_{\Omega} \nabla c_{\varepsilon} \cdot \nabla \varphi-\int_{0}^{\infty} \int_{\Omega} \frac{1}{\varepsilon}\left(\log \left(1+\varepsilon n_{\varepsilon}\right)\right) c_{\varepsilon} \varphi \\
& -\int_{0}^{\infty} \int_{\Omega} u_{\varepsilon} \cdot \psi_{t}-\int_{\Omega} u_{0} \cdot \psi(\cdot, 0)-\int_{0}^{\infty} \int_{\Omega} Y_{\varepsilon} u_{\varepsilon} \otimes u_{\varepsilon} \cdot \nabla \psi \\
& =-\int_{0}^{\infty} \int_{\Omega} \nabla u_{\varepsilon} \cdot \nabla \psi+\int_{0}^{\infty} \int_{\Omega} n_{\varepsilon} \nabla \psi \cdot \nabla \Phi
\end{aligned}
$$

hold. Now, since the dominated convergence theorem implies that

$$
\frac{1}{1+\varepsilon n_{\varepsilon}} \rightarrow 1 \text { in } L_{\mathrm{loc}}^{4}\left([0, \infty) ; L^{4}(\Omega)\right) \text { as } \varepsilon=\varepsilon_{j} \searrow 0
$$

the convergences of $n_{\varepsilon}$ in $L_{\text {loc }}^{2}\left([0, \infty) ; L^{2}(\Omega)\right)$ and $\nabla c_{\varepsilon}$ weakly in $L_{\text {loc }}^{4}\left([0, \infty) ; L^{4}(\Omega)\right)$ (see Lemma 4.1) derive

$$
\frac{n_{\varepsilon}}{1+\varepsilon n_{\varepsilon}} \nabla c_{\varepsilon}=n_{\varepsilon} \cdot \frac{1}{1+\varepsilon n_{\varepsilon}} \cdot \nabla c_{\varepsilon} \rightarrow n \nabla c \quad \text { weakly in } L_{\text {loc }}^{1}\left([0, \infty) ; L^{1}(\Omega)\right)
$$


as $\varepsilon=\varepsilon_{j} \searrow 0$. On the other hand, to confirm convergence of $\frac{1}{\varepsilon}\left(\log \left(1+\varepsilon n_{\varepsilon}\right)\right) c_{\varepsilon}$ in $L_{\mathrm{loc}}^{1}\left([0, \infty) ; L^{1}(\Omega)\right)$ we shall show that $f_{\varepsilon}\left(n_{\varepsilon}\right) \rightarrow n$ in $L_{\mathrm{loc}}^{2}\left([0, \infty) ; L^{2}(\Omega)\right)$ as $\varepsilon=\varepsilon_{j} \searrow 0$, where $f_{\varepsilon}(s):=\frac{1}{\varepsilon} \log (1+\varepsilon s)$ for $s \geq 0$. Noticing from (4.2) that

$$
\left|f_{\varepsilon}(n)-n\right|^{2} \rightarrow 0 \quad \text { a.e. in } \Omega \times(0, T) \text { as } \varepsilon=\varepsilon_{j} \searrow 0
$$

and from the inequality $f_{\varepsilon}(s) \leq s(s \geq 0)$ that

$$
\left|f_{\varepsilon}(n)-n\right|^{2} \leq 2 n^{2}
$$

we deduce from the dominated convergence theorem that for all $T>0$,

$$
\left\|f_{\varepsilon}(n)-n\right\|_{L^{2}\left(0, T ; L^{2}(\Omega)\right)}^{2}=\int_{0}^{T} \int_{\Omega}\left|f_{\varepsilon}(n)-n\right|^{2} \rightarrow 0
$$

as $\varepsilon=\varepsilon_{j} \searrow 0$. Therefore we can see from the inequality

$$
0<f_{\varepsilon}^{\prime}(s)=\frac{1}{1+\varepsilon s} \leq 1
$$

and (4.2) that for all $T>0$,

$$
\begin{aligned}
\left\|f_{\varepsilon}\left(n_{\varepsilon}\right)-n\right\|_{L^{2}\left(0, T ; L^{2}(\Omega)\right)} & \leq\left\|f_{\varepsilon}\left(n_{\varepsilon}\right)-f_{\varepsilon}(n)\right\|_{L^{2}\left(0, T ; L^{2}(\Omega)\right)}+\left\|f_{\varepsilon}(n)-n\right\|_{L^{2}\left(0, T ; L^{2}(\Omega)\right)} \\
& \leq f_{\varepsilon}^{\prime}\left(n_{\varepsilon}\right)\left\|n_{\varepsilon}-n\right\|_{L^{2}\left(0, T ; L^{2}(\Omega)\right)}+\left\|f_{\varepsilon}(n)-n\right\|_{L^{2}\left(0, T ; L^{2}(\Omega)\right)} \\
& \rightarrow 0
\end{aligned}
$$

as $\varepsilon=\varepsilon_{j} \searrow 0$. This together with (4.3) enables us to obtain that

$$
\frac{1}{\varepsilon}\left(\log \left(1+\varepsilon n_{\varepsilon}\right)\right) c_{\varepsilon}=f_{\varepsilon}\left(n_{\varepsilon}\right) c_{\varepsilon} \rightarrow n c \quad \text { in } L_{\mathrm{loc}}^{1}\left([0, \infty) ; L^{1}(\Omega)\right)
$$

as $\varepsilon=\varepsilon_{j} \searrow 0$. Then all convergences in Lemmas 4.1, 4.2 as well as (4.11), (4.12) make sure to pass to the limit in all integrals in (4.8)-(4.10), which means that the triplet $(n, c, u)$ is a global weak solution of (1.1).

\section{References}

[1] X. Cao. Global bounded solutions of the higher-dimensional Keller-Segel system under smallness conditions in optimal spaces. Discrete Contin. Dyn. Syst., 35:18911904, 2015.

[2] X. Cao, S. Kurima, and M. Mizukami. Global existence and asymptotic behavior of classical solutions for a 3D two-species chemotaxis-Stokes system with competitive kinetics. Math. Methods Appl. Sci., to appear, arXiv:1703.01794 [math.AP].

[3] Y. Chung and K. Kang. Existence of global solutions for a chemotaxis-fluid system with nonlinear diffusion. J. Math. Phys., 57:19 pp, 2016.

[4] N. Dunford and J. T. Schwartz. Linear Operators. I. General Theory. Pure and Applied Mathematics, 1958. 
[5] T. Hashira, S. Ishida, and T. Yokota. Finite-time blow-up for quasilinear degenerate Keller-Segel systems of parabolic-parabolic type. J. Differential Equations, to appear.

[6] T. Hillen and K. J. Painter. A user's guide to PDE models for chemotaxis. J. Math. Biol., 58:183-217, 2009.

[7] M. Hirata, S. Kurima, M. Mizukami, and T. Yokota. Boundedness and stabilization in a two-dimensional two-species chemotaxis-Navier-Stokes system with competitive kinetics. J. Differential Equations, 263:470-490, 2017.

[8] D. Horstmann and G. Wang. Blow-up in a chemotaxis model without symmetry assumptions. Eur. J. Appl. Math., 12:159-177, 2001.

[9] S. Ishida, K. Seki, and T. Yokota. Boundedness in quasilinear Keller-Segel systems of parabolic-parabolic type on non-convex bounded domains. J. Differential Equations, 256:2993-3010, 2014.

[10] S. Ishida and T. Yokota. Blow-up in finite or infinite time for quasilinear degenerate Keller-Segel systems of parabolic-parabolic type. Discrete Contin. Dyn. Syst. Ser. $B, 18: 2569-2596,2013$.

[11] C. Jin. Boundedness and global solvability to a chemotaxis model with nonlinear diffusion. J. Differential Equations, 263:5759-5772, 2017.

[12] E. F. Keller and L. A. Segel. Initiation of slime mold aggregation viewed as an instability. J. Theor. Biol., 26:399-415, 1970.

[13] J. Lankeit. Eventual smoothness and asymptotics in a three-dimensional chemotaxis system with logistic source. J. Differential Equations, 258:1158-1191, 2015.

[14] J. Lankeit. Long-term behaviour in a chemotaxis-fluid system with logistic source. Math. Models Methods Appl. Sci., 26:2071-2109, 2016.

[15] J. Lankeit and Y. Wang. Global existence, boundedness and stabilization in a highdimensional chemotaxis system with consumption. Discrete Contin. Dyn. Syst., 37:6099-6121, 2017.

[16] N. Mizoguchi and M. Winkler. Blow-up in the two-dimensional parabolic Keller-Segel system. preprint.

[17] T. Nagai, T. Senba, and K. Yoshida. Application of the Trudinger-Moser inequality to a parabolic system of chemotaxis. Funkcial. Ekvac., 40:411-433, 1997.

[18] K. Osaki, T. Tsujikawa, A. Yagi, and M. Mimura. Exponential attractor for a chemotaxis-growth system of equations. Nonlinear Anal., 51:119-144, 2002.

[19] K. Osaki and A. Yagi. Finite dimensional attractor for one-dimensional Keller-Segel equations. Funkcial. Ekvac., 44:441-469, 2001. 
[20] J. Simon. Compact sets in the space $L^{p}(0, T ; B)$. Ann. Mat. Pura Appl., 146:65-96, 1987.

[21] Y. Tao and M. Winkler. A chemotaxis-haptotaxis model: the roles of nonlinear diffusion and logistic source. SIAM J. Math. Anal., 43:685-704, 2011.

[22] Y. Tao and M. Winkler. Boundedness in a quasilinear parabolic-parabolic KellerSegel system with subcritical sensitivity. J. Differential Equations, 252:692-715, 2012.

[23] Y. Tao and M. Winkler. Global existence and boundedness in a Keller-Segel-Stokes model with arbitrary porous medium diffusion. Discrete Contin. Dyn. Syst., 32:19011914, 2012.

[24] M. Winkler. Aggregation vs. global diffusive behavior in the higher-dimensional Keller-Segel model. J. Differential Equations, 248:2889-2905, 2010.

[25] M. Winkler. Boundedness in the higher-dimensional parabolic-parabolic chemotaxis system with logistic source. Comm. Partial Differential Equations, 35:1516-1537, 2010.

[26] M. Winkler. Global large-data solutions in a chemotaxis-(Navier-)Stokes system modeling cellular swimming in fluid drops. Comm. Partial Differential Equations, 37:319-351, 2012.

[27] M. Winkler. Finite-time blow-up in the higher-dimensional parabolic-parabolic Keller-Segel system. J. Math. Pures Appl., 100:748-767, 2013.

[28] M. Winkler. Global asymptotic stability of constant equilibria in a fully parabolic chemotaxis system with strong logistic dampening. J. Differential Equations, 257:1056-1077, 2014.

[29] M. Winkler. Global weak solutions in a three-dimensional chemotaxis-Navier-Stokes system. Ann. Inst. H. Poincaré Anal. Non Linéaire, 33:1329-1352, 2016.

[30] J. Zheng and Y. Wang. Boundedness and decay behavior in a higher-dimensional quasilinear chemotaxis system with nonlinear logistic source. Comput. Math. Appl., 72:2604-2619, 2016. 